\title{
A Bridge Dual-Character Time-Perceptive Traditionalized synergic Ranking Design for POI Proposal
}

\author{
Mrs. ${ }^{1}$ K.M.Priya' Mr. ${ }^{2}$ V.Gnanasekar, M.E, Mr. C.Suresh M.E \\ ${ }^{I}$ Pursuing M.E CSE Dept, ${ }^{2}$ Head of the department Computer Science and Engineering, ${ }^{3}$ Assistant professor \\ Dept of Computer science, Gojan School of Business and Technology, Redhills, Chennai.
}

\begin{abstract}
Tourism has become an important domain for most of the economies, especially for nonindustrialized countries where it represent the main source of income. Recommendation systems are the techniques which predict the rating of users interest with item based or society based entity. These items can be places, books, movies, restaurants and things on which individuals have different preferences. These preferences are analyzed and processed using two approaches first content-based filtering which involves distinctiveness of an item and second collaborative filtering approaches which takes into account user's precedent activities to make choice. Point of interest recommendation engine provides a feasible method for personalized recommendation of various places to its users.POI systems are generally more tedious in its operation comparatively to the already existing merchandise recommendation engines. The reason being the amount of time it takes for the services involved. When we consider the online services, designing a prominent approach for efficient and effective recommendation is very important. Generally, in the already existing services for searching and recommending the services are done by the keyword-based search which leads to the poor recommendation performance and leads to vast dependence on complex queries from the user. This proposal has advantage of functionality and competence, and thus possible for the realistic and good result with effective cost benefits.
\end{abstract}

\section{INTRODUCTION}

In today's fast growing technology trend, there's an enormous amount of data being shared across the web. This enormous data information has paved the growth of big data domain. The big data is in general a large diverse set of information that has been evolved from different sources. These sources define that they have their own volume, speed and variety of information that has been acquired.

After exploring through this huge collection of data from various sources the main difficulty involves in classify the amount of information from the large data sets. With the large amount of services in the web today, along the various computing and cloud computing services there has been enormous amount of data loss and issues in processing huge data's. As a result, service-related data become very difficult to effectively process by traditional approaches. With all the difficulties mentioned previously, a novel approach such that Clustering based collaborative filtering approach[8]which is one common method for recommendation, is adopted in this paper, which not only collect the similar interest in one cluster but also group the common interested item from the past. Thereby, recommending most interested and also popular item to the customer.

Recommendation system in this concept provides a personalized solution for user based on social and geographical influence. The information about the user is obtained through various social networking sites while their geographical information's are obtained from their most recent check-in data's. The suggestion provided by the system as to be more efficient that it has to capture the user's interest in their social influence.

However, this approach may fail when the user moves away from the check-in location [1]. Thus we cannot completely rely on the check-in data to obtain the user location information. To overcome the challenges faced by the already existing system that depends on check-in data we propose the POI system which overcomes the data-sparsity issue by covering both the user-based recommendation and item-based recommendation. Using the collaborative filtering we will work on the clusters, observe the similarity of user's interest on the location searched by producing the most relevant and efficient recommendation.

In this paper, we propose a point of interest recommendation system using hierarchical clustering based collaborative filtering technique to produce the user-based and Item based recommendation [2]. The user based 
recommendation will cover the most interested places whereas the item-based recommendation will have the popular search history. We designed the system with the Hierarchical clustering algorithm which observes the users rating and recommend the places in two ways. One is user based recommendation which has most interested places and the other one is item based recommendation which has the most visited history. This is one difference from all the existing system which generally captures reviews and just shows up with recommendation.

In relevant research, recommendation system works is often treated it's based on LBSN and the user interest explicitly provided by the various online social users[2].In this paper, we categorize the systems by user rating to compute the user-based clustering in addition to reviews of the system to the location visited earlier. Second, we form a cluster to group all the similar interested ratings. Third, we classify the POI system as user-based recommendation which are most interested place and item-based recommendation.

. For each category, we summarize the contributions of the recommendation work done and highlight the representative work. Additionally, we discuss about the data sets used for rating and the similarity metrics used for computation. Finally, we project the absolute possible future outcomes of the paper.

Cluster-based recommendation is best chosen method for user-based recommendation. Instead of recommending places to users, places are recommended to clusters of similar users. This entails a preprocessing phase, in which all users are partitioned into clusters. Each cluster will have the users with similar interest. Interest is captured in this POI system by means of rating the most visited and liked places. The cluster will have the most liked places and the popularity based location. Finally to produce the recommendation to the user, similarity coefficient is considered and calculated to capture most top rated places along with that of popularity based recommendation.

\section{RELATED WORK}

Mohammad Aliannejadi[1], has proposed a algorithm which is CR based for POI Recommendation and involves in the CR modeling based on explicit user feedback and ranking method using implicit user feedback in order to produce the point of interest recommendation. The recommendation is analyzed based on the geographical similarity between POI and also considering the user preference. Since this method focuses on check-ins data for ranking, which may not be very efficient and there arouse data sparsity problem.

Collaboration filtering is a technique used in most of the recommendation system today, which produces the recommendations based on user preferences. Xiwang yang with their members [2], presented a recommendation system of online social voting based on collaboration filtering technique .Online social networks, as become the wide range of social information sharing place for most of the users. In social networking site, asking for opinion or casting an online social voting as been the recent trend in these years. Despite all the technology growths designing an approach for online voting model as unique challenges and opportunities. Addressing all these challenges, a novel RS model was proposed which includes matrix-factorization (MF) based and nearest Neighbor (NN) based model to learn the user rating interest. In this paper, collaborative filtering based RS user feedback used to predict user interest, which leads to accurate recommendation. RS based model traces both social network information and group affiliation information to be mined which improves the popularity based recommendation.

Bin Liu and members [3], proposed geographical probabilistic model for point of interest (POI) advice which provides personalized advice of places, such as restaurant and movie theaters. There's been increasing popularity in the usage of mobile devices and LSBN's in recent trends which hold significant new opportunities as well as several challenges to be addressed. Understanding this concept influence major factors such as personal preferences, check-in data's, geographical factors and behavior of user mobility. While there are still studies which address the challenges in POI recommendation which fails to analyze large volumes of joint effect of multiple parameters. To overcome these problems, a general geographical probabilistic factor model (GeoPFM) framework was developed which strategically takes various factors into consideration. Particularly, this paper addresses the geographical influence using check-in data. Also, mobility model can be grasped effectively in POI Recommendation. Hence in this paper, based on Geo-PFM framework, Poisson based Geo-PFM design was proposed which provides a most exact probabilistic generative process for the entire model and is effective in modeling the distorted user check-in count data as unspoken feedback for better POI recommendations.

(C) 2020, IJSMS 
Point of interest (POI) recommendation has become a most popular way to provide customized recommendation to its user. When providing location based recommendation, there are several challenges like data sparsity, improper recommendation of data, user poi matrix and cold start issue. Hongzhi yin and members [4], proposed a paper, where a spatial aware hierarchical collaborative deep learning model has been adopted which overcomes the problem in spatial dynamic personal preferences. Cold start problem one of the critical issues in POI system, which indicates that the user as not used check-ins or not rated the system. In order to address the cold start problem and data sparsity issues non linear fusion method is used.

With the development of various recommendation systems in the market, filtering based techniques have been in usage for quite some time. Various filtering techniques involves Collaboration based filtering, Content based filtering, demographic filtering and hybrid filtering. Among mentioned techniques, collaboration based technique have been established most successful and implemented widely in the Recommendation system. In this paper, Mohammed wasid[5] with Vaibhor Kant proposed fuzzy-PSO CF based logic, which provides most accurate results in comparison with the real time. The results of this system involves most accurate binary logic(True or False). The system involves in finding the optimal priorities by collecting users different features such as age, gender, rating etc. With all the available parameters weights will be calculated for each and then similarities between parameters are calculated. A particle swarm algorithm is used along with CF approach to develop the recommendation system in three phases. Phases include user profile formation, neighborhood set generation, prediction and recommendation.

\section{PROPOSED WORK}

\section{PROPOSED ALGORITHM}

In this proposed system, Hierarchical clustering Algorithm with collaborative filtering to compute the personalized location based recommendation for users. Clustering is a technique, which involves in grouping of objects of similar kind. Here the user's similar interest (such as rating) has been clubbed together in single cluster. So similar interested user rating is available in one single cluster. To this cluster, collaborative filtering is applied which recommend the places to the user of their interest and with most popular demand. Similarity required for recommendation is calculated based on the highest value pair between two most liked places.

In the existing methods of recommendation the check-in data is considered as an important parameter to compute the interested place for recommendation. Also the main evolution in the proposed recommendation system, here it suggests both the liked and popular places to its user. It is evident that when the user moves to a different location, there's a data sparsity problem and we may not consider this location for recommendation.

Here, as in other recommender system we use CF based Hierarchical clustering method to group and recommend the user's with their interested places. Collaborative filtering (CF) could also be a way hired with the aid of recommender structures. Filtering the similarity among the items available using rating given by the user is called as item based collaborative filtering. This type of filtering suits best in the applications where users are more in number when compared with the items. Since users will give more rating to the items as well and it gets a stable rating distribution. Once the user purchases a product and rates it that item will be filtered and added to the user recommendation.

$$
\operatorname{pred}(u, i)=\overline{\mathrm{r}}_{\mathrm{u}}+\frac{\sum_{\mathrm{veN}} \operatorname{sim}(\mathrm{u}, \mathrm{v})\left(\mathrm{r}_{\mathrm{vi}}-\overline{\mathrm{r}}_{\mathrm{v}}\right)}{\sum_{\mathrm{v} \in \mathrm{N}} \operatorname{sim}(\mathrm{u}, \mathrm{v})}
$$

Where $\mathrm{N}$ denotes the nearest neighbour set of user $\mathrm{u}, \mathrm{r}_{\mathrm{v}, \mathrm{i}}$ denotes the rating of items $\mathrm{i}$ given by user $\mathrm{v}$, $r_{u}, r_{v}$ denotes the average rating given by the user $u$ and user $v$ respectively, $\operatorname{sim}(u, v)$ is the person coefficient between user $\mathrm{u}$ and user $\mathrm{v}[10]$. 
The following figure shows how the collaborative filtering works in mined user's details and shows the neighbouring formation using clusters

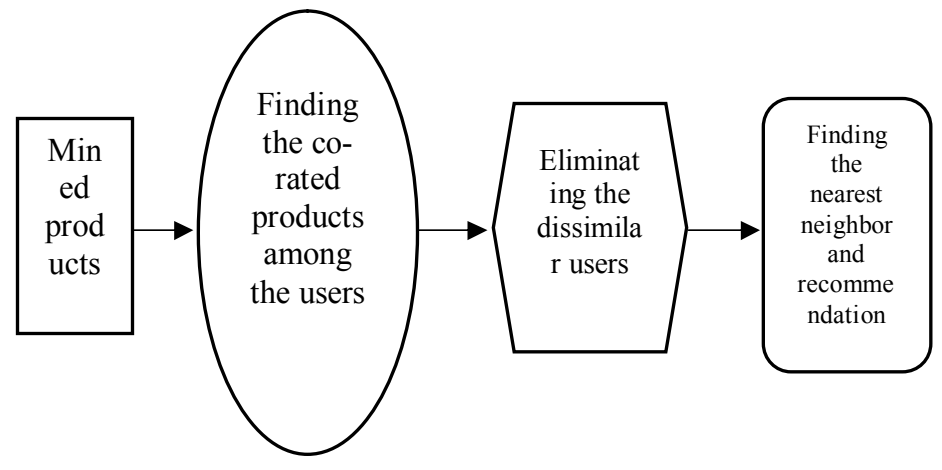

FIG1: COLLOBORATIVE FILTERING APPROAACH

\section{ARCHITECTURE DIAGRAM}

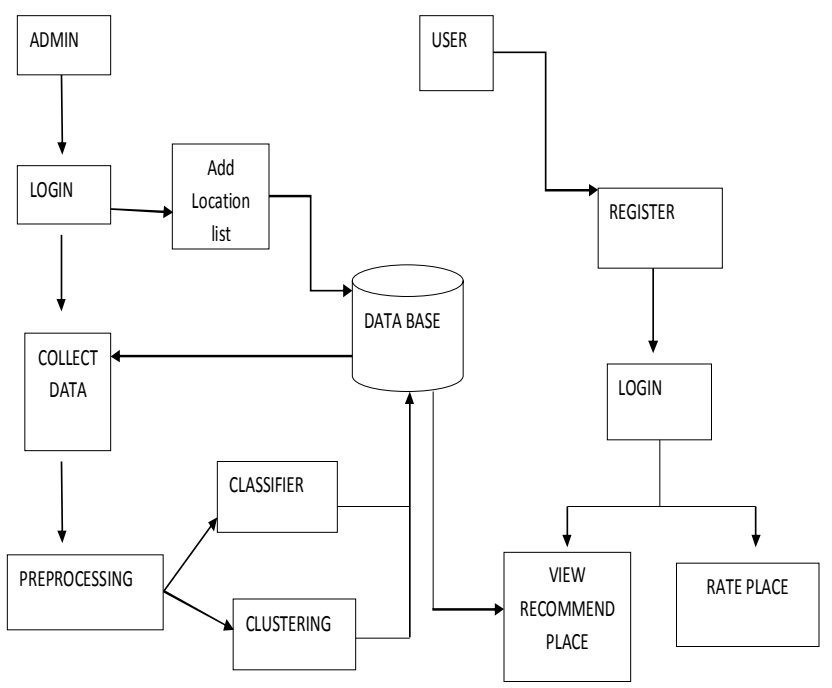

FIG 2: PROPOSED ARCHITECTURE

Above figure shows the proposed architecture of POI recommendation system. The POI recommendation system involves in two phase recommendation to the user. As shown in the architecture, the user can register and login into the system. In the system, they can search for new places, rate the already visited places and get the recommendations from the system, view the overall places visited or new places in global recommendation Map. To rate the places, the user can check the information in the rate places tab, where the user is prompted with places to be rated is prompted. User can also view overall rating provided by other users, which gives the idea of nearest and most relevant places to be viewed. Based upon these ratings, recommendations are provided to users in two stages. The ratings captured from the user are processes, compared with other user's rating for similarity ratio. The similarity ratio involves nearest popular place, mostly liked places and ratings of the users. The two stage Recommendation is then presented to the user which is Item based and User based. The Item based recommendation will have the most popular places to visit whereas the User based recommendation will have the places that most liked and interested by the user. 


\section{PROPOSED MODULES}

The proposed POI recommendation system consists of following modules and functionalities.

\section{REGISTRATION WITH POI SYSTEM:}

The POI system provides the most user friendly access to the users by means of user login registration and admin roles. The new user must use the POI system after the successful completion of registration with the basic details such as Id, Latitude and longitude, Age. Similarly the Admin portal has the predefined access to the system with admin id and password which can be used to process the data during preprocessing. Once the Username and password is created user can login into the POI system and check for the required information on the places and sightseeing.

\section{PREPARATION OF DATA}

The dataset used for this study was prepared from the tourist places Repository. Here we having the three data sets are location, users and rating. The location data attributes include country name, city name, place name and the user data including the attributes are user id, age, gender and password. The rating dataset having the attributes are user id, place name and ratings. Once the data has been gathered such as rating and user login related information datasets will be created accordingly. Gathered data were clustered to process it separately for the effective analysis. Once the clustering process is completed, data is pre-preprocessed to check the efficiency and sustainability of the received information from user.

\section{LOCATION DETAILS}

In this module admin can add new places with details and their location details. These details will add to the existing details. User can select the place details added in this module and they will rate the place based on their reviews. These details are used for cluster the data based on their ratings. User can update the location details of the previous selection.

\section{DATA PRE PROCESSING}

The training data, we are given a list of vectors $(u ; m ; r ; t)$, where $u$ is a user ID, $m$ is a places name; $r$ is the rating $\mathrm{u}$ gave to $\mathrm{m}$, and $\mathrm{t}$ is the date. After training, application output predictions for a list of user-item pairs. Application measure error by using the root means squared error. After pre-processing, application the places name with the corresponding users and their ratings with separated files.

\section{DATA CLUSTERING}

Group based suggestion is best idea as a variation on client based proposal. Rather than prescribing things to clients, things are prescribed to groups of comparable clients. This involves a pre-handling stage, in which all clients are parceled into bunches. Recommendations are then created for each group, with the end goal that the prescribed things are most fascinating to the biggest number of clients. The upside of this approach is that proposal is quick at runtime on the grounds that nearly everything is precompiled. One could contend that the proposals are less individual along these lines, since suggestions are processed for a gathering as opposed to a person. This approach might be more compelling at delivering proposals for new clients, who have little inclination information accessible. In this sense, it is important to consider that clusters are formed according to the similarities and dissimilarities between the analyzed countries. Therefore tourism professionals and researchers should consider that the identity and consciousness of the people.

\section{POI RECOMMENDATION}

POI Recommendation system is a personalized engine which provides recommendation or suggestion for the data users based upon their search. In this paper, recommendation is provided to user in two stages. One is userbased recommendation which was produced by calculating the most rated places by the number of users. The second is item-based recommendation in which most popular place is suggested to the user. Both the recommendations are highly efficient with most likely places and popularity based. In this POI system, agglomerative clustering used which helps in clustering similar liked placed users in order to provide only the 
liked and most popular places to the users. And also the visited places are shown to the user with Map added to the POI design using the API.

\section{PERFORMANCE METRICS}

Applications like data analytics, statistics generally involves in processing large amount of data. Each datasets corresponds to collection of data, variables or separate database attributes. In such situation it is very essential to analyze the performance of the various attributes.

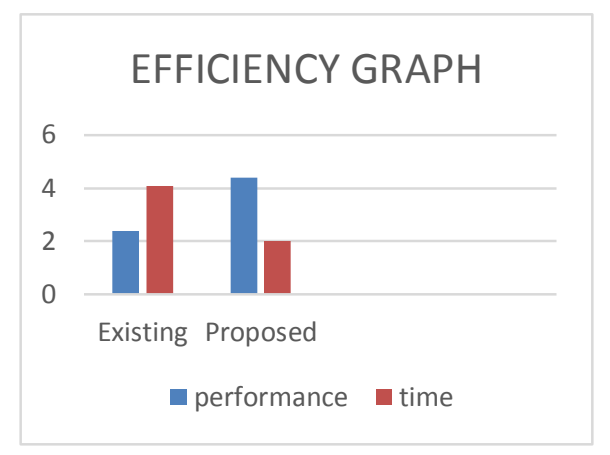

FIG 3: PERFORMANCE GRAPH

As shown in the graph considering the performance against the time we could see that the proposed system grows better in its performance with respect to the efficiency for recommending the relevant information the user interested. It is very important to recommend the most popular places to user which is also interested and liked by the same user.

\section{CONCLUSION AND FUTURE WORKS}

Big data brings great expediency for people. Predominantly, it perfectly matches the increased need of data usage. In this paper, to build an efficient recommendation system in cost effective and more relevant way we present an Agglomerative Hierarchical clustering approach for big data applications relevant to service recommendation. A cluster of services has been formed using AHC algorithm to which $\mathrm{CF}$ is applied. Then the similarities between the ratings are computed within the same cluster. The Club CF costs less computation time as the number of services in the cluster is small when compared with the whole system. The Ratings of services in the same cluster are more accurate with each other than with the ones in other clusters. If we want, add the new places with name, city and country. These datasets will be preprocessed by admin and users. The recommendation will be provided in two phases, popularity based and user based. Also the clear picture of places visited is presented to the user by means of location Map. This proposal has advantage requisites of functionality and competence, and thus is possible a realistic and producing good result with effective cost benefit.

In considering some of the improvements to this paper as future works, the user ratings can be managed efficiently furthermost using the user's social media interferences such as instagram, facebook and twitter sites to capture the live ratings and additionally reviews could also be captured. When considering the reviews, we can think about the document similarity ratio for visited places description and user comments. Then applying clustering based collaborative filtering will yield a best and relevant recommendation to the user's who are interested in travel.

\section{REFERENCES}

[1] Mohammed Aliannejadi,Dimtrios Rafailidis and Fabio Crestani ,"A Joint two phase Time-sensitive Regularized Colloborative Ranking Model for Point of Interest Recommendation”IEEE transaction on knowledge and Data Engineering /TKDE2019

[2] Xiwang Yang, Chao Liang, Miao Zhao, Hongwei Wang, Hao Ding, Yong Liu, Yang Li, and Junlin Zhang “colloborative filteringbased recommendation of online social voting”,2017 
[3] Bin Liu, HuiXiong, Senior Member, IEEE, Spiros Papadimitriou, Yanjie Fu, and Zijun Yao "A general geographical probabilistic factor model for point of interest recommendation,in IEEE,2015

[4] H. Yin, W. Wang, H. Wang, L. Chen, and X. Zhou, "Spatial-aware hierarchical collaborative deep learning for POI recommendation," IEEE Trans. Knowl. Data Eng., vol. 29, no. 11, pp. 2537-2551, 2017.

[5] Mohammed wasid and vibhor kant,"A particle swarm approach to collaborative filtering based Recommender system through fuzzy features",2015(IMCIP-2015).

[6] M. Aliannejadi, I. Mele, and F. Crestani, "Personalized ranking for context-aware venue suggestion," in SAC, 2017, pp. 960-962.

[7] J. Bao, Y. Zheng, D. Wilkie, and M. F. Mokbel, "Recommendations in location-based social networks: a survey," GeoInformatica,vol 19 , no. 3, pp. 525-565, 2015.

[8] M.C pham,Y.Cao,R.Klamma,et al,"A Clustering Approach for collaborative filtering recommendation using social network analysis"Journal of universal computer science,Vol 17,No.4,PP.583-604,April 2011

[9] V. W. Zheng, Y. Zheng, X. Xie, and Q. Yang, "Collaborative location and activity recommendations with GPS history data," in WWW, 2010

[10] B. M. Sarwar, G. Karypis, J. A. Konstan, and J. Riedl, "Item-basedcollaborative filtering recommendation algorithms," in WWW,2001, pp. 285-295.

[11] C. Zhang, H. Liang, and K. Wang, "Trip recommendation meets real-world constraints: POI availability, diversity, and traveling time uncertainty,” ACM Trans. Inf. Syst., vol. 35, no. 1, pp. 5:1-5:28,2016.

[12] L. Chen, G. Chen, and F. Wang, "Recommender systems based on user reviews: the state of the art," User Modeling and User-Adapted Interaction, vol. 25 , no. 2 , pp. $99-154,2015$ 